\title{
Steady-State Local Diffusive Fluxes in Porous Geo-Materials Obtained by Pore-Scale Simulations
}

\author{
Yoshito Nakashima · Tsukasa Nakano
}

Received: 5 September 2011 / Accepted: 27 February 2012 / Published online: 21 March 2012

(C) The Author(s) 2012. This article is published with open access at Springerlink.com

\begin{abstract}
Computer simulations of non-sorbing tracers diffusing in fluid-saturated porous sediment/rock were performed using pore-scale X-ray microtomographic images to reveal the following. (i) The histogram of the magnitude of the local diffusive flux vector obeys a unimodal log-normal distribution having a long positive tail. Simulations using model images were also performed to show that the flux broadening in large pores and the flux mixing at the pore network junctions are responsible for the log-normal shape. (ii) The simulation enabled us to directly visualize pore voxels with large and small fluxes, confirming the existence of transport pores and stagnant pores. Because of the unimodal nature, however, it was difficult to distinguish transport pores from stagnant pores using an objective threshold in the histogram. (iii) Another histogram of the flux vector component along the direction of the macroscopic concentration gradient was analyzed. A negative tail was found in the histogram, indicating that local counter diffusion exists in the porous geo-materials. However, the population and intensity of the counter diffusion fluxes are too small and weak to contribute to the overall diffusive transport across the porous media system. A long positive tail representing a large-flux diffusive pathway was also observed in the histogram. However, again, the population of the large-flux transport pores is small. As a result, the main conveyer of the tracer is the stagnant pores (not the transport pores), which have small positive flux values but a large population.
\end{abstract}

Keywords Archie's law · Electric conductivity · Diffusion coefficient · Formation factor · Resistivity $\cdot$ X-ray CT

Electronic supplementary material The online version of this article (doi:10.1007/s11242-012-9976-1) contains supplementary material, which is available to authorized users.

Y. Nakashima $(\bowtie) \cdot$ T. Nakano

National Institute of Advanced Industrial Science and Technology (AIST),

Central 7, Higashi 1-1-1, Tsukuba, Ibaraki 305-8567, Japan

e-mail: nakashima.yoshito@aist.go.jp 


\section{Introduction}

The diffusion is an important mode of tracer transport in porous media systems with small Péclet numbers in earth science (e.g., Nakashima 1995; Grathwohl 1998; Takeda et al. 2011). The tracer diffuses in the pore network having large pores (pools) and narrow throats (e.g., Roberts and Schwartz 1985; Lindquist et al. 2000) as illustrated in Fig. 1. Thus, pore-scale analysis is one of the useful approaches to understanding the diffusive transport in porous media. Computer simulations are useful in investigating the pore-scale diffusion process in geo-materials (e.g., Nakano 1995; Nakashima et al. 2004; Promentilla et al. 2009; Han et al. 2009; Sugiyama et al. 2010). Thus, we performed extensive simulations to understand steadystate non-sorbing tracer diffusion in fluid-saturated porous geo-materials. Three-dimensional (3D) pore-scale images of sandy sediments and sandstone having various porosities and degrees of the pore anisotropy were prepared by X-ray microtomography followed by image processing. We numerically solved the Laplace equations within these pore-scale domains with respect to the tracer concentration and calculated the local steady-state diffusive flux vector in the pore space.

In the present study, we focused on the following problems concerning the pore-scale statistics of the local diffusion flux vector in the complex porous geo-materials. (i) The local counter flow was already found for the Darcy flow (not diffusion) in porous media (Kutsovsky et al. 1996; Maier et al. 1998; Ogawa et al. 2001; Li et al. 2010). The local flux obeys the log-normal distribution for the random resistor network (de Arcangelis et al. 1985) and for the Darcy flow (Cenedese and Viotti 1996; Lebon et al. 1996). We examined whether the counter diffusive flux and log-normal distribution were realized with respect to the tracer diffusion in porous media without Darcy flow. (ii) According to the conventionally accepted concept (e.g., Pérez-Rosales 1982; Lever et al. 1985; Skagius and Neretnieks 1986; Nakashima 1995; Tiab and Donaldson 2004), percolated pore space can be divided into two regions (transport pores with large flux and stagnant pores with small flux) as expressed in Eq. (A1). However, to the best of our knowledge, the validity of this concept has not been directly confirmed through pore-scale simulations. We calculated the spatial distribution and statistical histogram of the magnitude of the local flux vector, and attempted to find the fraction of transport pores and stagnant pores for a few different threshold numbers. (iii) The conventionally accepted concept described above implies that the degree of the contribution to the overall tracer diffusive transport across the porous media system is small for stagnant pores and large for transport pores (e.g., Skagius and Neretnieks 1986; Tiab and Donaldson 2004). In order to examine this implication, we analyzed a different histogram (flux-weighed histogram) of the longitudinal flux component.

Fig. 1 Two-dimensional schematic diagram of the diffusion through the pore network. Some pools are connected by narrow throats. The local diffusion directions in the throats are indicated by black arrows. The direction of the macroscopic concentration gradient is shown by an orange arrow

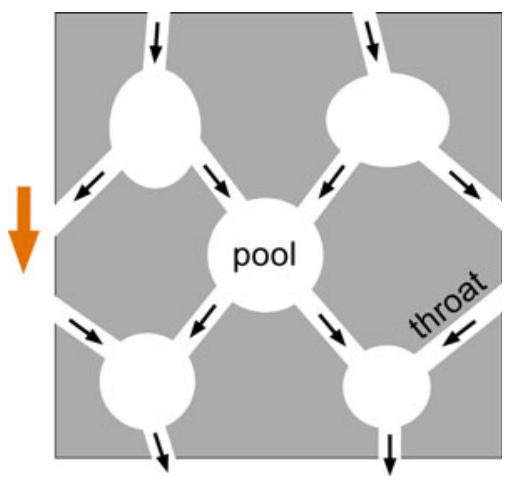




\section{Description of Diffusion Simulations}

The method to simulate the tracer diffusion in the 3D digital images of porous geo-materials and to analyze the obtained data on the local diffusive fluxes is outlined. The detail of the equations used is described in Appendix, and the symbol list and some remarks on the simulation programs are noted in ESM_1.pdf. First, the 3D pore-scale Laplace equation, Eq. (A2), was solved numerically by assuming the steady-state diffusion of non-sorbing species in fluid-saturated porous geo-materials (Zhang and Knackstedt 1995; Knackstedt et al. 2007; Øren et al. 2007; Zhan et al. 2010). The boundary conditions are such that a constant concentration difference was applied to the two opposite faces (normal to the $z$-plane), whereas no-flow boundary conditions are assumed on the other four faces. As a result, we obtained the $3 \mathrm{D}$ distribution of the tracer concentration for each pore voxel.

Second, we performed the following data analysis using the obtained concentration data, $C$. Based on Fick's first law, the local diffusive flux vector was calculated voxel by voxel by taking the spatial difference of $C$ :

$$
(J x, J y, J z)^{T}=-D_{\text {bulk }} \operatorname{grad} C
$$

where $J x, J y$, and $J z$ are the $x$-, $y$-, and $z$-components of the local flux vector of the pore voxels, respectively, and $T$ refers to the transposing operation. In the program of the present study, the operation of taking the spatial derivative in Eq. (1) was replaced with taking the finite difference of the concentration between adjacent voxels. Note that the diffusion coefficient in Eq. (1) is not $D_{\text {eff }}$, but rather $D_{\text {bulk }}$. We assumed that the direction of the macroscopic concentration gradient imposed on the 3D image system was the $z$-direction. Thus, $J z$ summed over the entire $x-y$ plane at a fixed $z$ position is proportional to $D_{\text {eff }}$ of the fluid-saturated porous media. The quantity $D_{\text {bulk }}$ is a given or known parameter of the system. The formation factor can then be calculated by substituting the $D_{\text {eff }}$ and $D_{\text {bulk }}$ values into Eq. (A3). As a result, we obtain the distribution of the flux vector $(J x, J y, J z)$ and its magnitude, $J=\left(J x^{2}+J y^{2}+J z^{2}\right)^{1 / 2}$ as $3 \mathrm{D}$ images, and the formation factor as a scalar. The obtained histogram of $J$ was fitted to a log-normal distribution:

$$
N=\frac{k_{1}}{\sqrt{2 \pi} k_{2} J} \exp \left(-\frac{\left(\ln J-k_{3}\right)^{2}}{2 k_{2}^{2}}\right) \quad \text { for } J>0
$$

where $N$ is the number of pore voxels, and $k_{1}, k_{2}$, and $k_{3}$ are parameters.

The $J z$ histogram (i.e., plot of $N$ as a function of $J z$ ) is useful in detecting the local counter flow having a negative $J z$ value. However, the $J z$ histogram is not appropriate to quantitatively evaluate the degree of the contribution of pore voxels having various $J z$ values. For example, the degree of the contribution to the overall diffusive transport across the image system of a single voxel of $J z=100$ (arbitrary unit, au) is equivalent to that of fifty voxels of $J z=2$, and to four voxels of $J z=25$ (i.e., $1 \times 100=50 \times 2=4 \times 25$ ). In order to avoid this undesirable underestimation of large-flux voxels, a flux-weighed histogram (i.e., plot of $N \times J z$ as a function of $J z$ ) was calculated.

Although solving Eq. (A2) to obtain steady-state local diffusive flux data is the main simulation in the present study, a different diffusion simulation was also performed as a supplement. This simulation is a random walk of non-sorbing tracers in the percolated pore cluster to calculate the normalized apparent diffusivity of Eq. (A4). The details of the random walk simulation are described elsewhere (Nakashima and Kamiya 2007; Nakashima et al. 2008).

The methods of the diffusion simulation and of data analysis described above was applied to $512^{3}$-voxel microtomographic images of four real geo-materials: Kinugawa sand (Fig. 2a), 


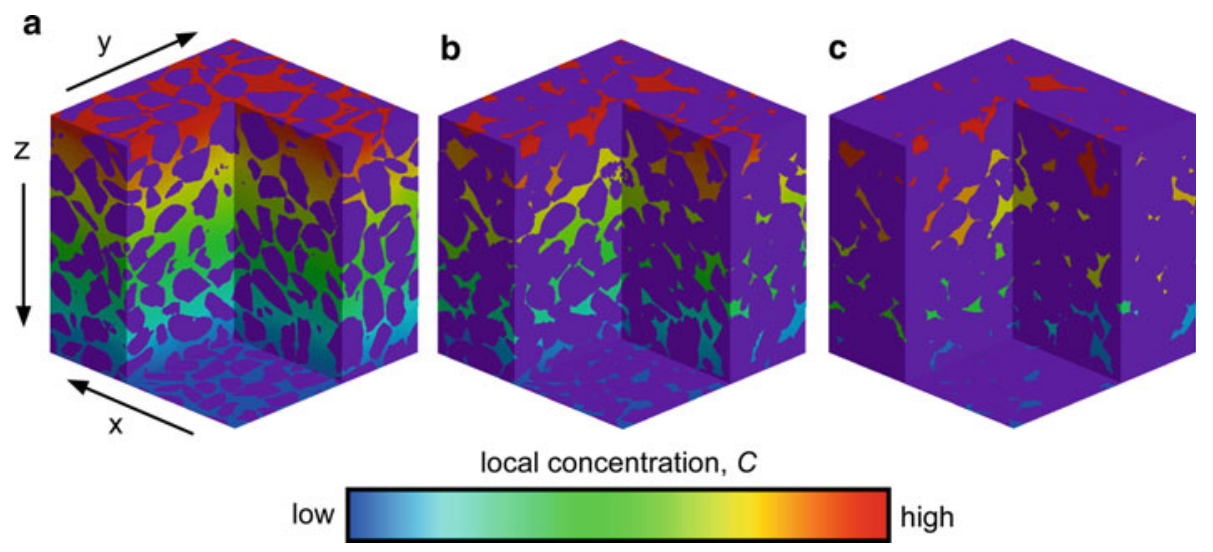

Fig. 2 Kinugawa sandy sediment images with various porosities without affine transformation. The dimensions of each image are $512^{3}$ voxels $=4.4^{3} \mathrm{~mm}^{3}$. Solid areas are indicated in purple. The computed local concentration, $C$, is superimposed on the percolated pore space. The macroscopic concentration gradient was applied along the $z$-axis. a Original image, porosity 41 vol $\%$. b Slightly pore-eroded image, porosity 18 vol\%. c Significantly pore-eroded image, porosity 8 vol\%

Kagoshima sand (Fig. ESM_1_1), monosized bead (Fig. ESM_4_1), and Berea sandstone (Fig. 3a). The details of the image processings of the four geo-materials are described in ESM_1.pdf. Based on Fig. 1, two types of 3D model images were also investigated to help one interpret the simulation results for the real microtomographic images. One model (Fig. 4) consists of a straight narrow cylindrical pipe (throat) and a large spherical pore (pool). This throat-pool structure is a characteristic pore structure of sandstones and sandy sediments (e.g., Roberts and Schwartz 1985). The other model is a random network of equiradii pore pipes without pools (Fig. 5). Numerous cylindrical straight pipes having a tortuosity of unity are connected randomly to mimic the pore channel network having a specific coordination number at the network junctions in natural sedimentary rocks (Lindquist et al. 2000). Affine transformation and pore-erosion operation were applied to the two models. The Laplace equation for the pore space of Figs. 4 and 5 was solved to analyze the statistics of the local flux vector for comparison with the results for real sediment/sandstone images.

\section{Results}

All the obtained values of the formation factor, diffusion coefficient, and porosity are crossplotted to confirm the reliability of the 3D images used (see ESM_2.pdf for detail). The $J$ distribution for a model image of Fig. 4 is shown as 3D images (Fig. 4) and histograms (Fig. 6). In Fig. 6, a peak appears at $J=205$ (au), but this is an artifact due to the truncation. All four distributions were confirmed to be unimodal by the full-scale plot of the $J$-axis. Thus, this artifact-derived undesirable peak is omitted for other histograms in the present study. Figure 4 shows important features of the diffusive flow in porous media. The flow injected from the inlet (throat) into the spherical or spheroidal pool diffuses in the broad space, and the magnitude of the local flux vector decreases in the pool. The comparison with the original structure (Fig. 4a) and the affine-transformed or pore-eroded structure (Fig. 4bd) demonstrates that (i) the small-flux region appears due to the image compression in the $z$-direction and due to the pore erosion, and (ii) the large- $J$ region becomes more dominant by the image elongation along the $z$-direction. The features also appear in the $J$-histogram 
a
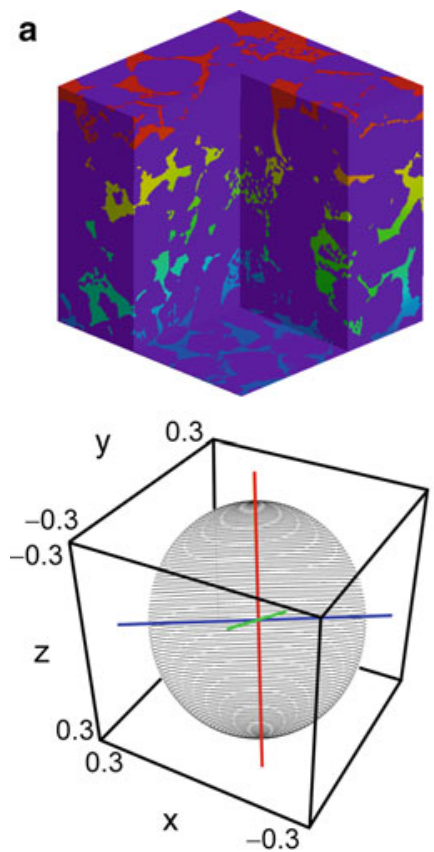
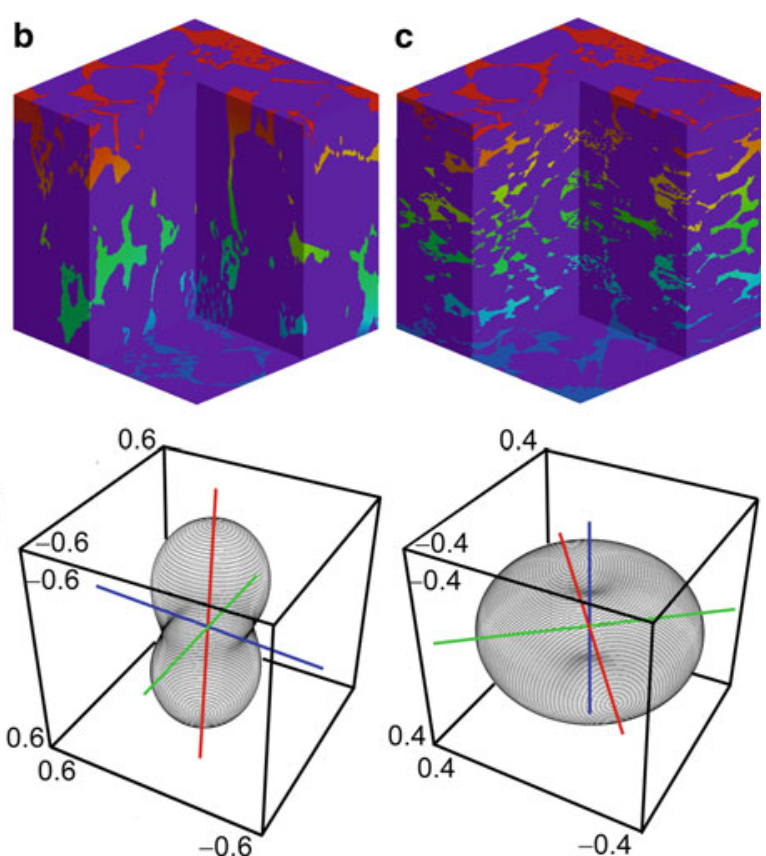

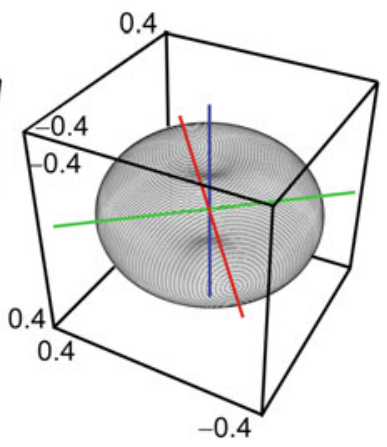

Fig. 3 Same as Fig. 2, but for Berea sandstone having various degrees of the affine transformation. The wireframe rendering of the normalized direction-dependent apparent diffusivity is also shown. The red, green, and blue lines indicate the major, intermediate, and minor axes of the diffusion tensor, respectively. a Original image $(\phi=20 \mathrm{vol} \%)$. The image dimensions are $512^{3}$ voxels $=0.64^{3} \mathrm{~mm}^{3}$. b Uniaxially elongated along the $z$-axis by a factor of two $(\phi=19 \mathrm{vol} \%)$. c Uniaxially compressed along the $z$-axis by a factor of one half $(\phi=20 \mathrm{vol} \%)$. A mirrored image of the half-compressed image was merged at $z=256$ to obtain a cubic system of $512^{3}$ voxels

(Fig. 6). The peak positions of the histograms for Fig. 4b-d shift leftward, leftward, and rightward, respectively. The log-normal distribution, Eq. (2), was reasonably fitted to the four histograms for $0<J<205$.

Results for another model image are shown in Figs. 5 and 7. The magnitude of the flux vector differs from pipe to pipe due to the difference of the 3D pipe position in the pore network (Fig. 5). The mixing of the diffusive fluxes having different $J$ values occurs at each pipe junction. As a result, the broad distribution having a long tail (i.e., log-normal distribution) similar to that in Fig. 6 appears in Fig. 7. The effects of the pore cementation (Fig. 5b) and image compression/elongation (Fig. 5c, d) on the peak shift of the log-normal distribution of Fig. 7 are the same as those of Fig. 6. These log-normal behaviors observed for the model images of Figs. 4 and 5 also appeared in the microtomographic images of the real geo-materials, which are shown later in Figs. 9a and 11a.

Some simulation results related to the 3D distribution of the local tracer concentration are shown in Figs. 2 and 3. The local concentration gradually varies along the direction of the macroscopically applied concentration gradient, i.e., the $z$-axis. Examples of the normalized direction-dependent apparent diffusivity are shown in Fig. 3. The shape of the shell-like representation of the apparent diffusivity is sensitive to the pore anisotropy induced by the uniaxial elongation/compression along the $z$-axis. The maximum-to-minimum ratio of the eigen-values of the diffusion tensor exceeds a critical value of 3/2 (Nakashima and Kamiya 


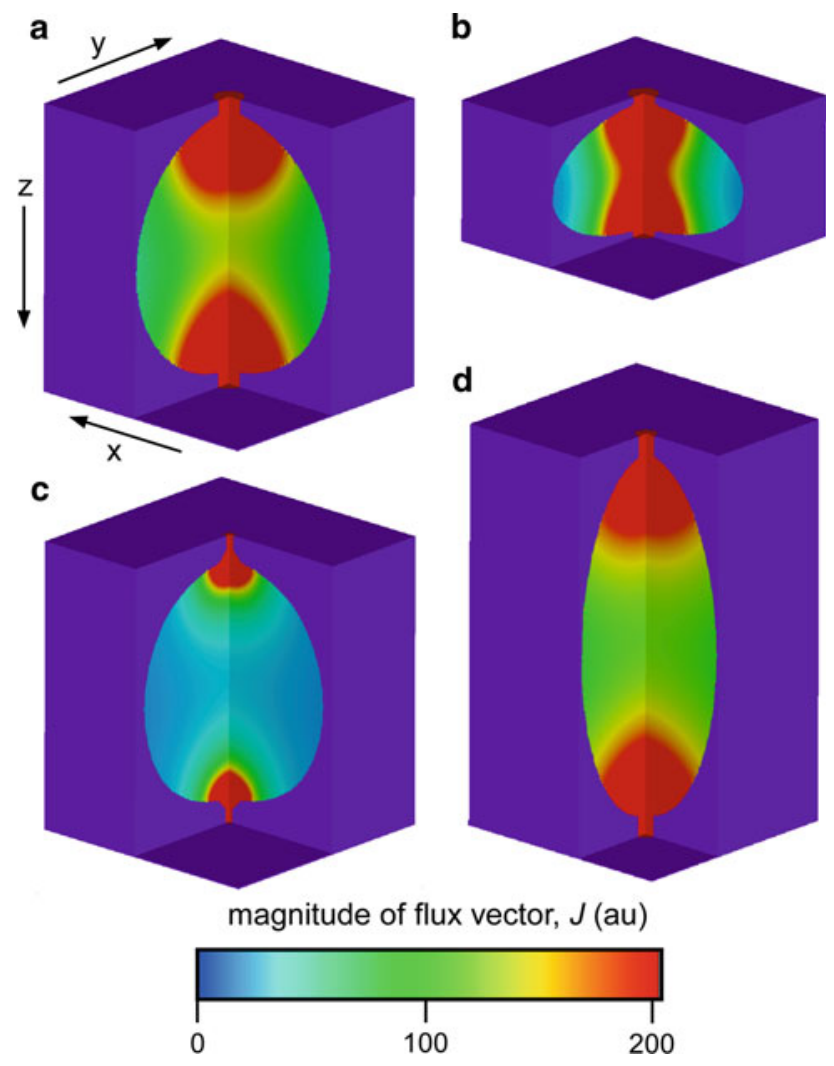

Fig. 4 Model having a throat-pool structure. Solid areas are indicated in purple. The magnitude of the computed local diffusive flux vector, $J$, is superimposed on the pore space. The macroscopic concentration gradient was applied along the $z$-axis. a The diameters of the cylindrical throat and spherical pool are 21 and 181 voxels, respectively. b Longitudinally compressed by a factor of one half to obtain an oblate pool. The throat diameter remains as 21 voxels. c Pore erosion or solid dilation was performed to obtain a throat diameter of 7 voxels. d Longitudinally elongated by a factor of two to obtain a prolate pool. The throat diameter remains as 21 voxels

2010) for the elongated and compressed images. Thus, the shape of the normalized directiondependent apparent diffusivity is no longer convex, but a constriction appears in Fig. 3b, c.

Typical examples of 3D images of the local diffusive fluxes for real geo-materials are shown in Fig. 8 for the Kinugawa sand images of Fig. 2a, c. Two-dimensional examples for the Kagoshima sand are also shown in Fig. ESM_3_1 to show the effects of the porosity reduction on the local diffusive flux more clearly. Figure 8 indicates that the spatial distribution of the flux is very complicated and that both stagnant (Fig. 8c, g) and percolated large-flux (Fig. 8b, f) zones exist. The growth of the green stagnant zones having small- $J$ values with decreasing porosity is shown by the 2D images (Fig. ESM_3_1a-d). Note also that the local counter flux occurs and grows with decreasing porosity (blue voxels in Fig. 8d, $\mathrm{h}$ and ESM_3_1e-h). As for the Kinugawa sand images of Fig. 2a, c (i.e., Fig. 8a, e), the decrease in porosity from 41 to 8 vol\% yields the increase in the formation factor value from 4 to 211 (Fig. ESM_2_1a) according to Archie's law. This increase in the macroscopic quantity of the formation factor is a consequence of the significant microscopic change in the local fluxes as shown in Fig. 8. 

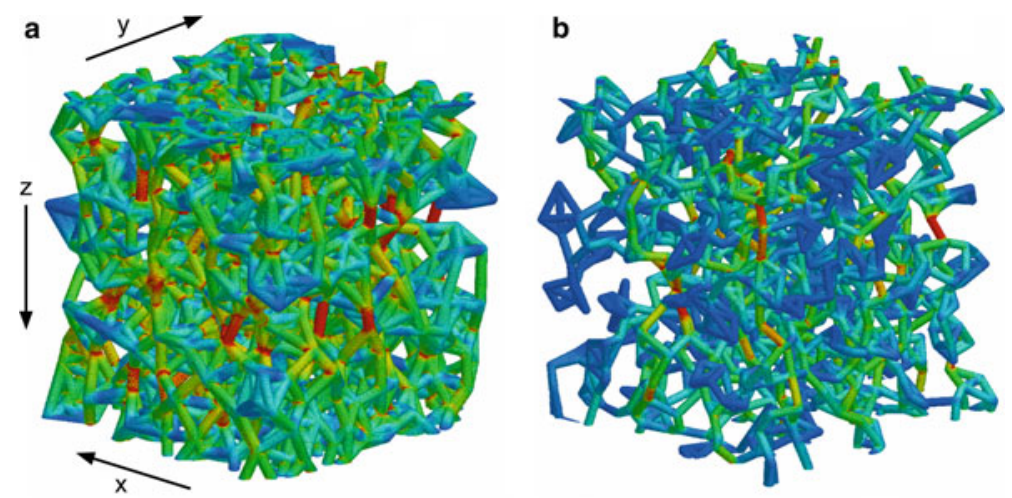

C
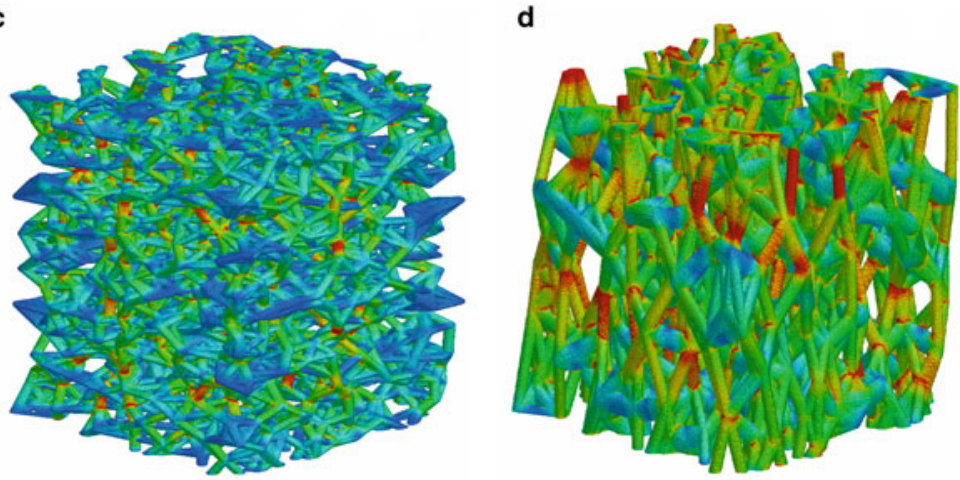

magnitude of flux vector, $J(\mathrm{au})$

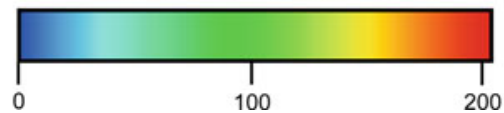

Fig. 5 Random network model of equiradii cylindrical pore pipes. The image dimensions are $500^{3}$ voxels. Solid voxels are made transparent. The calculated $J$ value is superimposed on the pore space. a The diameter of the cylindrical pipe is 17 voxels, the characteristic coordination number of pipes at each junction is 6 , $\phi=20 \mathrm{vol} \%$, and $F=12$. b The pipe diameter is 13 voxels, the characteristic coordination number is 3 , $\phi=7 \mathrm{vol} \%$, and $F=81$. The image $\mathbf{b}$ is a model of cementation of (a) obtained by reducing the coordination number and pipe diameter. c Obtained by the uniaxial compression of (a) along the $z$-axis by a factor of one half. $F=22$. d Obtained by the uniaxial elongation of (a) along the $z$-axis by a factor of two. $F=8$

The effects of the porosity on the statistics of the local diffusive fluxes are shown in Fig. 9 for the Kinugawa sandy sediments. Figure 9a indicates that the log-normal distribution fits the simulation data of $J$ well. Three parameters, $k_{1}, k_{2}$, and $k_{3}$ in Eq. (2) were determined by the least squares method in the log-normal fitting processing. The mode or distribution peak in Fig. 9a is systematically located at the left-side of the vertical dotted line, indicating that the diffusive flow in real geo-materials is significantly reduced compared to that in straight pores due to the tortuous pore structure having throats, pools, and various pore connectivities. The log-normal distribution is characterized by the dominant component of the stagnant regions having small $J$ values (e.g., Fig. 8c, g) and a long tail corresponding to the large $J$ components (e.g., Fig. 8b, f). However, it is difficult to objectively find a unique threshold of the $J$ value for discrimination between the stagnant and transport porosities because the obtained distribution of Fig. 9a is unimodal. For example, the threshold values for the 
Fig. 6 Histogram of the magnitude of the local diffusive flux vector, $J$, for each image, (a)-(d), in Fig. 4. The total number of pore voxels is adjusted to be the same between (a), (b), and (d). The data were fitted to the log-normal distribution, Eq. (2), by the least squares method
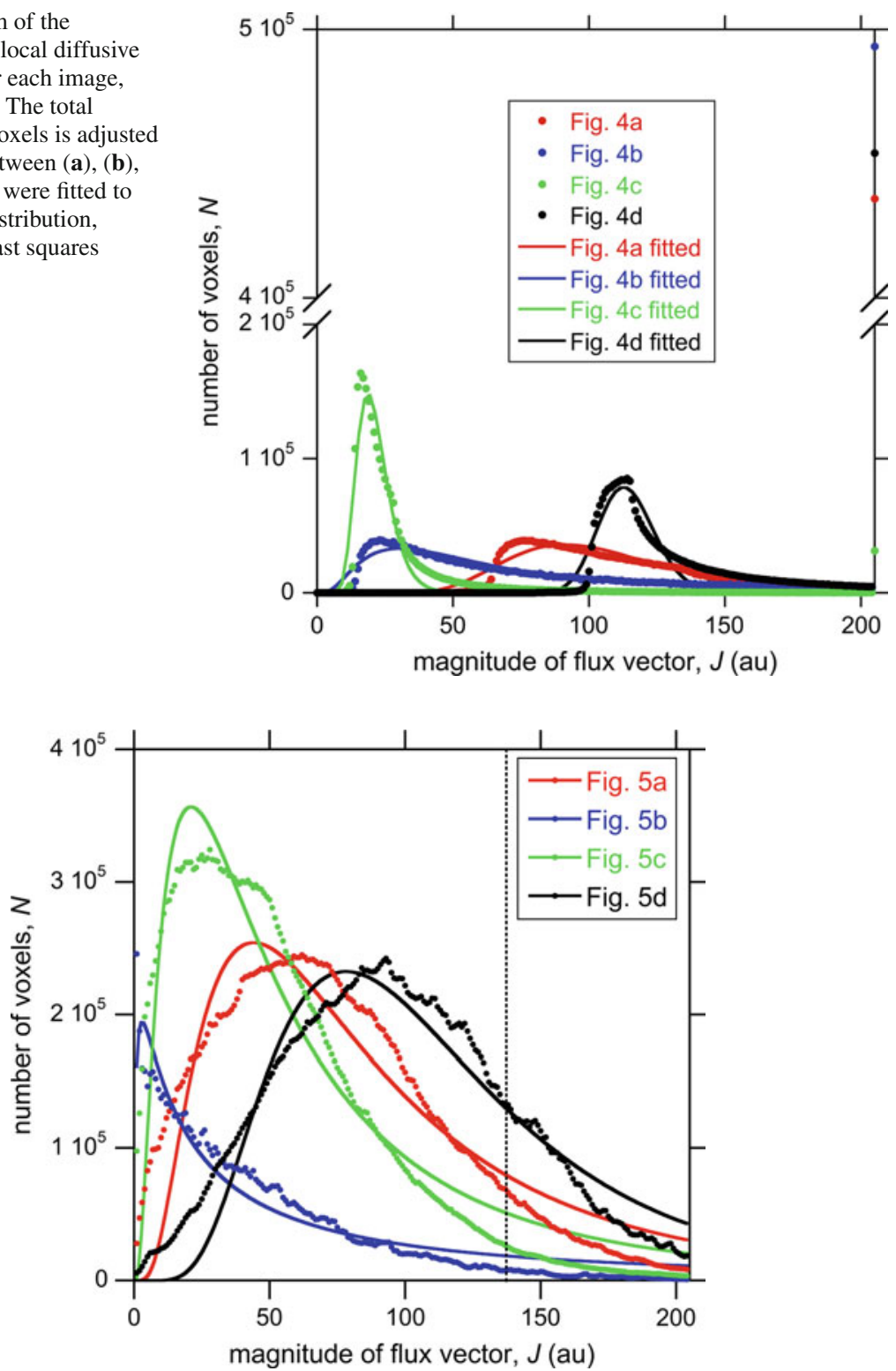

Fig. 7 Same as Fig. 6 but for the random pipe network of Fig. 5. The vertical dotted line indicates the $J$ value for the straight pipes completely parallel to the $z$-axis

percolation from the inlet of $z=0$ to the outlet of $z=511$ are $J=164$ and 27 for Fig. 8b, $\mathrm{f}$, respectively. Thus, it is possible to put a threshold for discrimination between the stagnant and transport pores to be $J=164$ and 27 in Fig. 8b, f, respectively, if one wishes to obtain the critical percolation state of the transport pores. However, there is no reasonable physical basis for choosing the percolation threshold of the large-flux pore voxels as the threshold for discrimination between the stagnant and transport porosities. Thus, in order to clearly show 
a

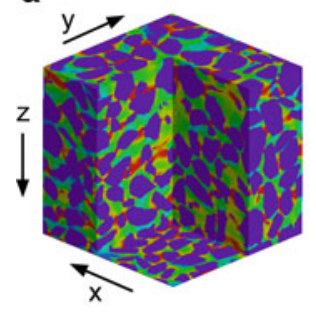

e

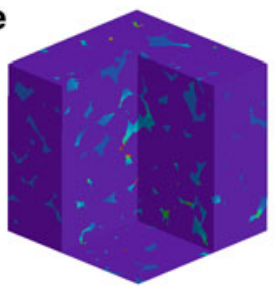

b

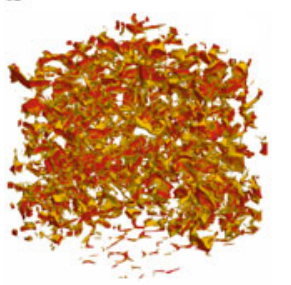

f

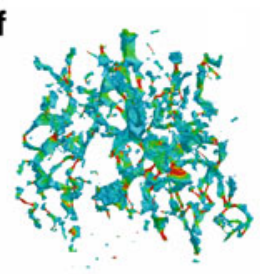

C

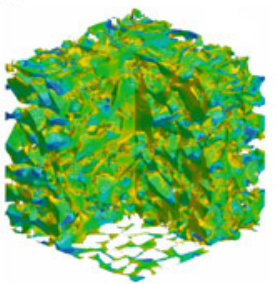

g

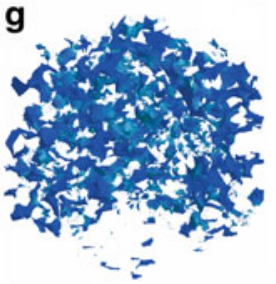

d

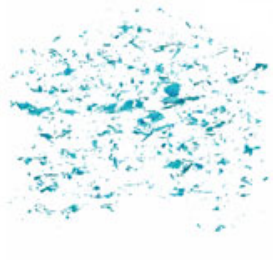

h

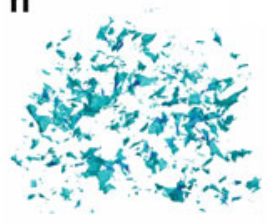

magnitude of flux vector, $J(a u)$

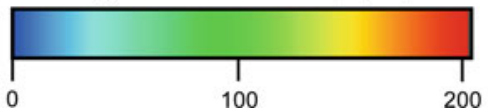

Fig. 8 Calculated local diffusive fluxes for Kinugawa sand images of Fig. 2a (a-d) and of Fig. 2c (e-h). The magnitude, $J(\mathbf{a}-\mathbf{c}$ and $\mathbf{e}-\mathbf{g})$ and $z$-component, $J \mathbf{z}(\mathbf{d}, \mathbf{h})$ of the local diffusive flux vector are shown. Solid areas in (a) and (e) are indicated in purple. Only pore voxels having large $J$ values $>160$ and 20 are visualized in (b) and (f), respectively, and voxels having small $J$ values equal to or $<160$ and 20 are visualized in (c) and (g), respectively. The thresholds of 160 and 20 were chosen tentatively. Only pore voxels having negative $J z$ values are shown in blue in (d) and (h). The graphic display condition for the view direction and image cutout is common to (a)-(h)

the simulation results in Figs. 8, 10, ESM_3_1, and ESM_6_1, different threshold values were tentatively used in the present study.

In Fig. 9a, the peaks shift leftward with decreasing porosity, indicating that the porosity reduction causes the stagnant regions to grow. This systematic growth of the stagnant regions having small $J z$ values caused by the porosity reduction also appears in Fig. 9b. The increase in pore tortuosity due to the pore reduction (Fig. ESM_2_1b) is probably responsible for the systematic growth. Figure $9 \mathrm{~b}$ indicates a small amount of local counter flux having negative $J z$ values, which is clearly confirmed by Fig. 9c. The population of the negative $J z$ increases almost systematically with decreasing porosity due to the increase in tortuosity.

The histogram of $N \times J z$ allows us to quantitatively evaluate the degree of the contribution of pore voxels having various $J z$ values. Figure $9 d$ clearly shows that the contribution of the local counter flow having a negative $J z$ value is negligible. The $J z$ values of the histogram peaks indicated by the arrows in Fig. $9 d$ are smaller than the value for the straight cylindrical pore having a tortuosity of unity, suggesting that the peaks correspond to the stagnant regions. Figure $9 \mathrm{~d}$ clearly shows that the stagnant regions are responsible for the main transport of the tracer across the system. The long positive tail corresponding to the transport pores having large $J z$ values expands significantly beyond the vertical dotted line (Fig. 9e). However, the population is small compared with that for each peak in the histogram. Thus, the long positive tail does not dominantly contribute to the tracer transport across the $512^{3}$-voxel system.

While Figs. 8, 9, and ESM_3_1 are used to examine the effects of the porosity reduction on the local diffusive flux, Figs. 10, 11, and ESM_6_1 are used to investigate the effects of the pore anisotropy. Figure 10 is the same as Fig. 8, except for the elongated (Fig. 10a-d) 

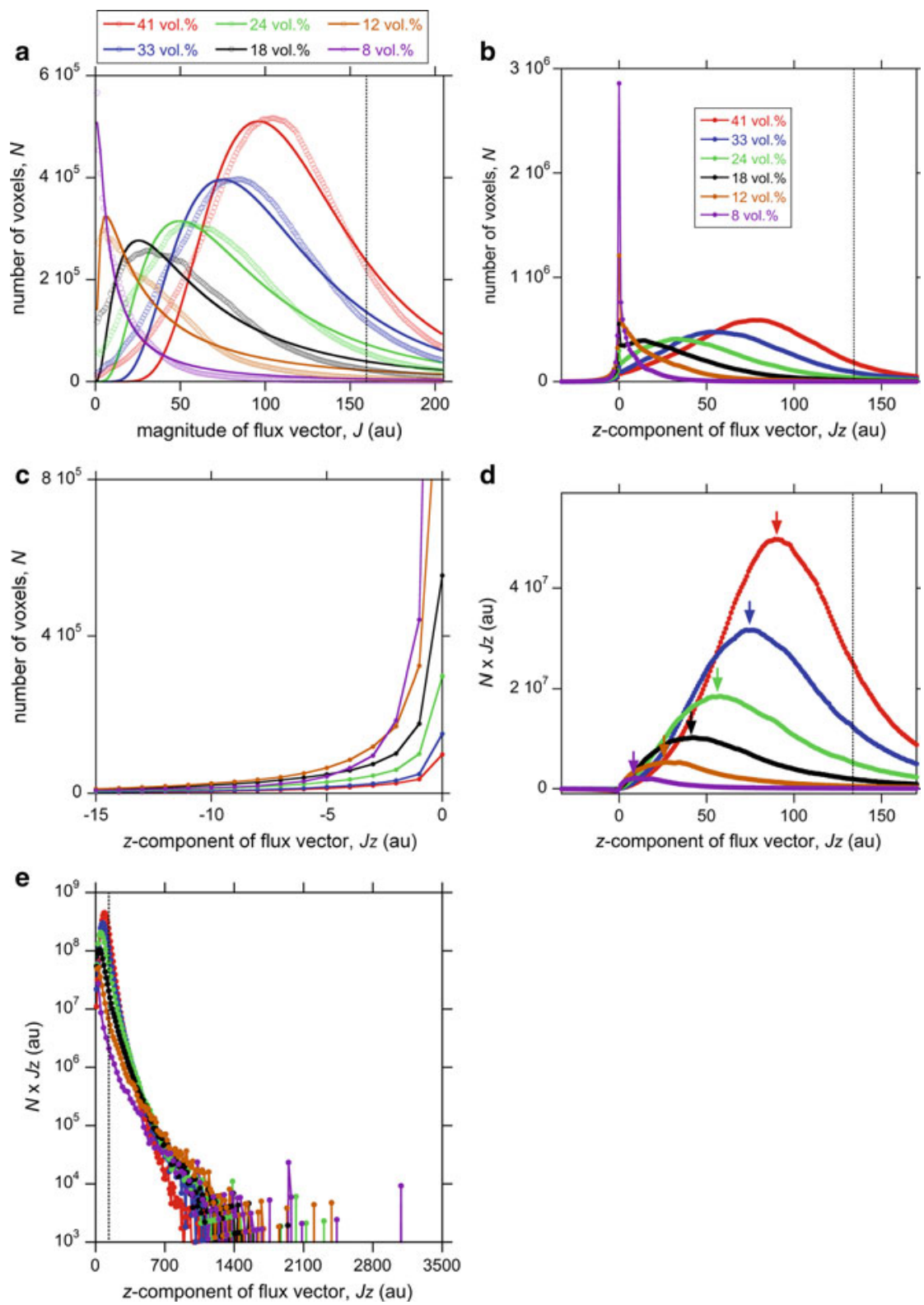

Fig. 9 Effects of the porosity on the histogram of the local diffusive flux vector for the Kinugawa sand images without affine transformation. The results for the images in Figs. 2 and 8 are also shown. The porosity values are indicated in vol\%. The legend is common to (b)-(e). The dotted line indicates the value for the straight pores that are completely parallel to the $z$-axis. A wider range was needed for the scaling of $J z$ having positive and negative values compared with that of $J$ having only positive values. As a result, the position of the dotted line is slightly different (i.e., 160 for (a) and 134 for (b, d, e)). a Magnitude of the flux vector. The log-normal distribution, Eq. (2), was fitted. The full-scale plot of (a) is shown in ESM_5.pdf. b $z$-component of the flux vector. c Magnification of the negative tail of (b). d Same as (b), but the vertical axis was modified to be the product of $N$ and $J$ z. The peak position of each distribution is indicated by a downward arrow. e Full-scale plot of the long positive tail of (d). Downward arrows in (d) are omitted. The bin size or column width of the histogram is different between (d) and (e), resulting in the difference in scale of the vertical axis 
a

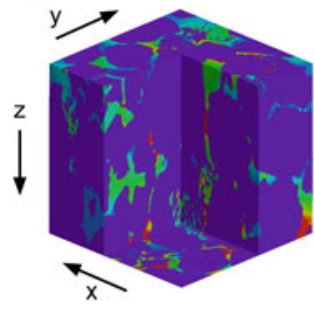

e

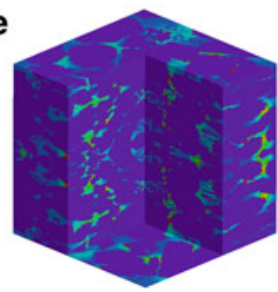

b
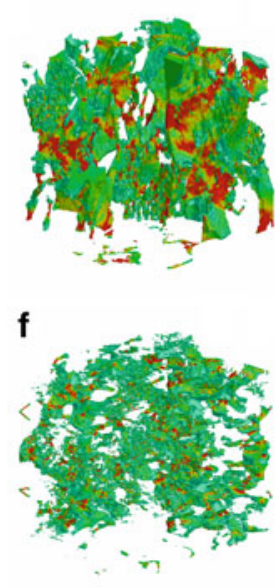

c
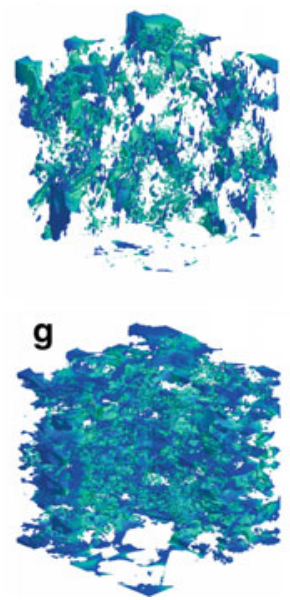

d

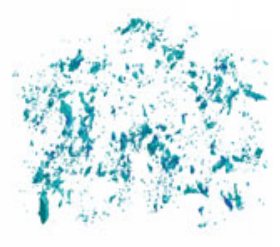

h

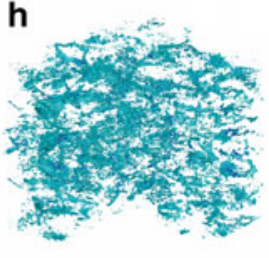

Fig. 10 Same as Fig. 8 but for Berea sandstone images of Fig. 3b (a-d) and of Fig. 3c (e-h). Only pore voxels having large $J$ values greater than a tentatively chosen threshold of 100 are visualized in (b) and (f). Voxels having small $J$ values equal to or $<100$ are visualized in (c) and (g)

and compressed (Fig. 10e-h) Berea sandstone images. Figure ESM_6_1 is the same as Fig. ESM_3_1, except for various degrees of the affine transformation of the bead pack images. The growth of the stagnant zones having small $J$ values with the change from elongation to compression is evident in Figs. 10 and ESM_6_1. The local counter flux exists and grows with the change from elongation to compression. Thus, the effects of the image compression along the $z$-axis have the same tendency as those of the porosity reduction due to the poreerosion operation. This tendency also appears in the histograms of Fig. 11. The degree of the fitting of Eq. (2) is somewhat poor in Fig. 11a compared with the sand pack images of Fig. 9a. For example, a very small second peak appears at $J \approx 0$, particularly for the two elongated images. However, Fig. 11a indicates that the log-normal distribution is mostly applicable to the natural sandstone images. The effects of the change from elongation to compression on the shape of the log-normal histogram of Fig. 11a have the same tendency as those of the porosity reduction of Fig. 9a. Figure 11b, c shows that both the stagnant component having small $J$ values and the negative tail of $J z$ contract in the elongated images and develop in the compressed images. The contribution of the negative tail of the $J z$ histogram to the overall tracer transport across the $512^{3}$-voxel system is negligible due to the small magnitude and small voxel number (Fig. 11d). The contribution of the long positive tail having large- $J z$ values to the overall tracer transport is small compared with the stagnant component having a high peak (indicated by the arrow in Fig. 11d) due to the small population (Fig. 11e).

\section{Discussion}

\subsection{Log-Normal Distribution}

Figures 9a (Kinugawa sand) and 11a (Berea sandstone) show that the magnitude of the local flux vector obeys the log-normal distribution. We have confirmed that the log-normal distribution is also applicable to two other samples, namely, the synthetic bead (Fig. ESM_4_3) and the Kagoshima sand. The changes in the porosity and in the degree of the 

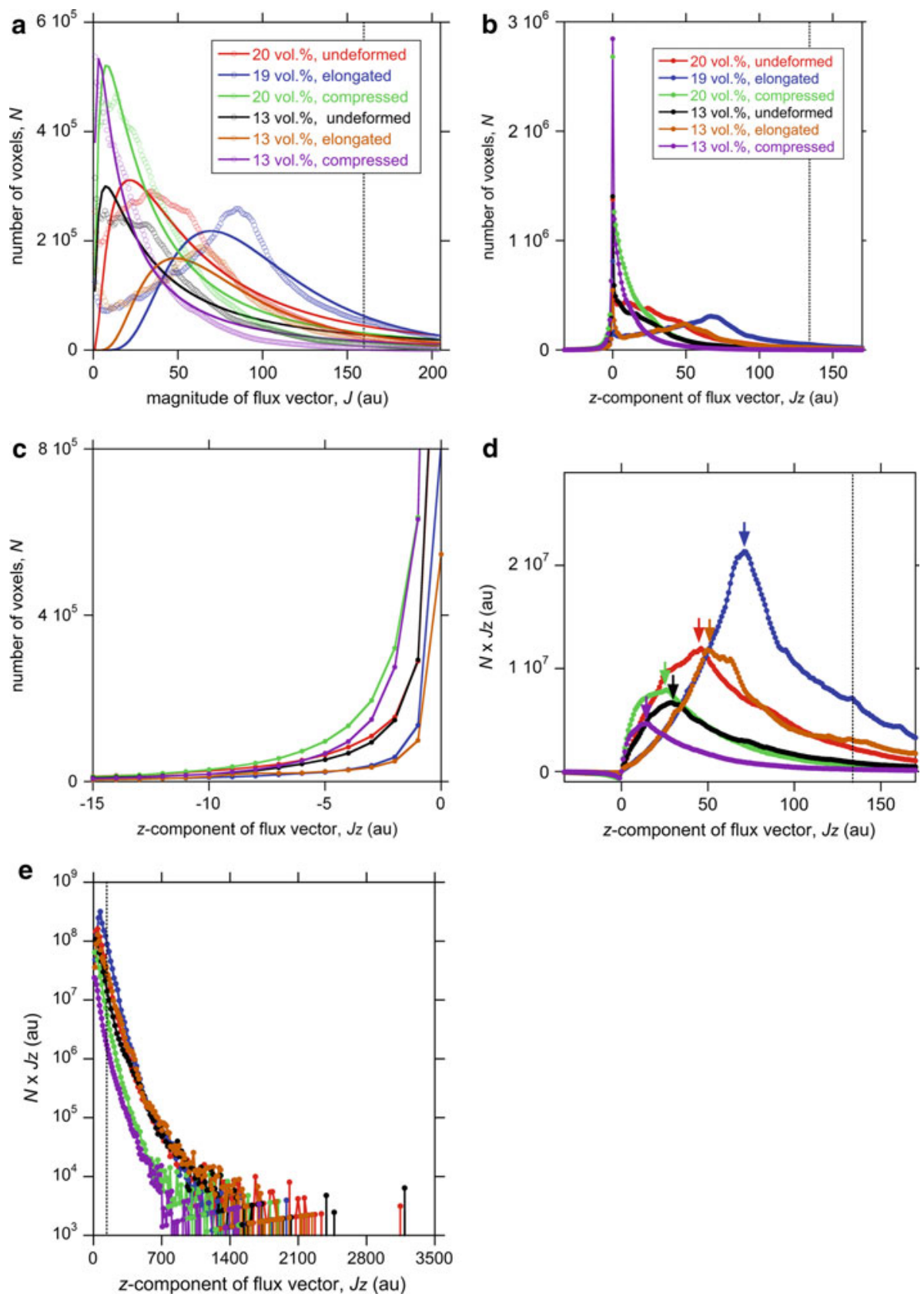

Fig. 11 Same as Fig. 9, with the exception of the effects of the degree of the affine transformation on the Berea sandstone images. The results for the images in Figs. 3 and 10 are also shown

affine transformation affect the tortuosity of the pore structure (Fig. ESM_2_1b), resulting in a significant change in the shape of the distribution. For example, the porosity reduction and image compression lead to the leftward shift of the histogram (Figs. 9a, 11a). The image 
elongation causes a rightward shift (Fig. 11a). Although the effects of the image deformation and porosity reduction on the macroscopic formation factor value have been discussed by a number of authors (e.g., Sen et al. 1981; Roberts and Schwartz 1985; Schön 2004; Nakashima and Nakano 2011), the present pore-scale study is the first systematic report on their effects on the microscopic statistics of the local diffusive/electric flux.

Based on the pore structure of Fig. 1, two model images of Figs. 4 and 5 were investigated to explore the mechanism that yields the log-normal distribution. We observed that the two models successfully reproduced the log-normal distribution. The diffusive flux having a large $J$ value was injected from the throat into the large pool in Fig. 4. Owing to the geometric contrast in pore diameter between the throat and the pool, the diffusive flux is broadened in the pool. This broadening is an inevitable outcome of the nature of the Laplace equation of Eq. (A2) and yields the log-normal distribution having a long positive tail shown in Fig. 6. Figure 5 depicts that the magnitude of the flux vector is different from pipe to pipe because the $3 \mathrm{D}$ pipe position in the pore network is different. The mixing of the diffusive flow having different $J$ values occurs at each pipe junction to yield a broad log-normal distribution of $J$. Note that an pipe system with equiradii can reproduce the log-normal distribution (pools are not essential). In conclusion, the flux broadening in large pores and the flux mixing at the pore network junctions are responsible for the log-normal distribution.

The log-normal distribution has been reported for the electric current in the random resistor network (de Arcangelis et al. 1985) and for the longitudinal velocity component for the Darcy flow in porous media (Cenedese and Viotti 1996; Lebon et al. 1996). The electric potential obeys the Laplace equation of Eq. (A2) as well as the tracer concentration. Thus, the electric current in the random network and the diffusive flux in the complex porous geo-materials would appear to obey the same log-normal distribution. On the other hand, the Darcy flow vector obeys a different equation, namely, the Stokes equation (e.g., Lebon et al. 1996) with a viscous boundary condition on the solid-fluid interface. Thus, it may appear strange that the diffusive flux and Darcy flux obey the same log-normal distribution. However, the local pore fluid pressure driving the Darcy flow obeys the Laplace equation as well as the concentration. The throat-pool structure (Fig. 4) and pore-network structure (Fig. 5) yield qualitatively the same effects (i.e., broadening and mixing of flows) on both the diffusive flow and Darcy flow driven by the concentration or pressure gradient. Thus, it is not surprising that the diffusive flux and the Darcy flux obey the same log-normal distribution.

\subsection{Negative Tail and Long Positive Tail}

The local counter diffusive flux, as well as the Darcy counter flow (Kutsovsky et al. 1996; Maier et al. 1998; Ogawa et al. 2001; Li et al. 2010), was observed in the present study (Figs. 9c, 11c). The porosity and pore anisotropy affect the population of the counter flux significantly. For example, the porosity reduction generally increases the population (Figs. 8d, h, 9c, and ESM_3_1e-h). The image elongation and compression decreases and increases the population, respectively (Figs. 10d, h, 11c, and ESM_6_1d-f). The diffusion is a scalar potential flow that obeys Fick's first law. Thus, the separation of flow at a high Reynolds number (e.g., Andrade et al. 1997; Watanabe and Katagishi 2006) cannot be responsible for the local counter diffusive flux. Rather, the complex tortuous pore geometry produced by the pore reduction and image compression (Fig. ESM_2_1b) is responsible for the generation of the counter flux.

Although the existence of the local counter flux is evident in Figs. 8, 10, ESM_3_1, and ESM_6_1, the magnitude and population are both small compared with the pore voxels having positive $J z$ values. As a result, the contribution of the negative tail to the overall 
transport of the tracer across the image system is negligible (Figs. 9d, 11d). This is consistent with Li et al. (2010), who reported that the contribution of the counter flow was insignificant for the Darcy flow in porous media.

The long positive tail corresponding to the transport pores having large $J z$ values was identified in the $J z$ histogram (Figs. 9b, 11b). Although it is difficult to find an objective threshold for the discrimination of the large- $J z$ voxels and stagnant voxels owing to the almost unimodal nature of the distribution, the $J z$ value of 134 (vertical dotted line in Figs. 9d, e, 11d, e) was chosen as a tentative threshold. Then, the contribution of all voxels having $J z$ values larger than the threshold to the total diffusive transport of the system can be calculated as the ratio of the area in the histogram. The ratio was as small as 13-19\% for the six cases of Fig. 9e and 13-24\% for those of Fig. 11e. This calculation demonstrates that the long positive tail corresponding to the transport pores shares a fraction of the tracer transport across the system, but its contribution is not the most dominant, and the stagnant pores are the main conveyer of the tracer.

\subsection{Concept of $\phi=\phi_{\text {trans }}+\phi_{\text {stag }}$}

The systematic Laplace simulations of the present study for the 3D images having various porosities and degrees of deformation revealed that a broad spectrum of diffusion flux values is realized in the $J$ and $J z$ histograms. It is possible to assign the large-flux pore voxels to $\phi_{\text {trans }}$ and the small-flux pore voxels to $\phi_{\text {stag }}$. The porosity reduction makes $\phi_{\text {stag }}$ more dominant than $\phi_{\text {trans }}$ (Fig. 9). While the image compression increases $\phi_{\text {stag }}$, the image elongation decreases $\phi_{\text {stag }}$ (Fig. 11). By properly choosing the threshold, it is possible to depict the percolated cluster of $\phi_{\text {trans }}$ having large-flux values, as demonstrated in Figs. 8b, f and 10b, f. In this sense, the conventional concept of $\phi=\phi_{\text {trans }}+\phi_{\text {stag }}$ is confirmed in the present study through pore-scale simulations.

However, this concept may give the impression that there is a distinct and objective threshold that distinguishes $\phi_{\text {trans }}$ and $\phi_{\text {stag }}$ in terms of the flux value. We found that this is not true because the histograms in Figs. 9 and 11 are almost unimodal due to the flux broadening in large pores and the flux mixing at the pore network junctions (Figs. 4, 5, 6, 7). As a result, it is difficult to find an objective threshold.

Most importantly, although the contribution of the transport porosity is not negligible, the stagnant porosity, $\phi_{\text {stag }}$, having relatively small flux values is the main conveyer of the tracer across the porous media system. Figures 9d, e and 11d, e clearly show that the peaks (indicated by arrows) that have smaller $J z$ values than the tentative threshold of the vertical dotted line significantly contribute to the overall tracer transport. This is a consequence of the number of pore voxels in the stagnant region being large enough to compensate for the small value of $J z$. Andrade et al. (1997) has reported that the stagnant region is important for the momentum transport of the Darcy flow in porous media. The present study revealed that the stagnant region is also important in the diffusive transport in porous media.

Because no Darcy flow was assumed in the simulations of the present study, the Péclet number of the porous media system studied is infinitesimal. The Péclet number would be finite for some real geological environments where the Darcy flow is not negligible. It is possible to qualitatively discuss the tracer transport with a finite Péclet number. Suppose that the Péclet number increases with the increase of the Darcy velocity. The tracer migrates in the porous system by the Fickian diffusion and Darcy flow, and the contribution of the latter would be more dominant with increasing flow magnitude. For a system with a sufficiently large Péclet number, the local tracer flux vector mostly agrees with the local Darcy flux vector. Then the histogram of the local tracer flux depends on the Reynolds number 
(Maier et al. 1998), the negligible local counter flux occurs (Kutsovsky et al. 1996; Ogawa et al. 2001; Li et al. 2010), and the $J z$ component obeys the log-normal distribution (Cenedese and Viotti 1996; Lebon et al. 1996). It should be also noted that the 3D migration trajectory of tracers dissolved in the flowing fluid would switch from the less tortuous path to more tortuous one having larger local pore diameters (Zhang and Knackstedt 1995).

\section{Conclusions}

Pore-scale diffusion simulations were performed for the non-sorbing tracers diffusing in the fluid-saturated porous sediment/rock to obtain the spatial distribution of the local diffusive flux vector. The main outcomes are as follows: (i) The histogram of the magnitude of the flux vector obeys a unimodal log-normal distribution. The flux broadening at large pores and flux mixing at pore junctions are responsible for the log-normal shape. (ii) The conventional understanding that pores consist of transport pores with large flux and stagnant pores with small flux was confirmed directly by the pore-scale simulation. Owing to the unimodal nature, however, it is difficult to distinguish transport pores and stagnant pores using an objective threshold. (iii) The local counter diffusion was observed. However, the population and the intensity of the counter fluxes are too small and weak to contribute to the overall diffusive transport across the porous media system. The population of the transport pores having large flux values was also too small to be the most dominant in the overall diffusive transport. As a result, the main conveyer of the tracer across the $3 \mathrm{D}$ image system is the stagnant pores having small positive flux values but a large population.

Acknowledgments Comments by three anonymous reviewers were very helpful. Mathematica $\mathrm{R}$ programming was performed by S. Kamiya. The Berea sandstone sample was imaged by Canon Marketing Japan Inc. using a MicroXCT system (Xradia, California, USA). Rock samples other than the Berea sandstone were imaged at the Japan Synchrotron Radiation Research Institute (Proposal No. 2001B0501-NOD-np) for the preliminary study. The present study was supported in part by JSPS KAKENHI (No. 23241012).

Open Access This article is distributed under the terms of the Creative Commons Attribution License which permits any use, distribution, and reproduction in any medium, provided the original author(s) and the source are credited.

\section{Appendix: Basic Equations Used}

The percolated pore porosity, $\phi$, in porous media can be divided into transport porosity, $\phi_{\text {trans }}$, having relatively large diffusive flux and stagnant porosity, $\phi_{\text {stag }}$, having relatively small diffusive flux (e.g., Tiab and Donaldson 2004):

$$
\phi=\phi_{\text {trans }}+\phi_{\text {stag }}
$$

The stagnant pores can comprise not only dead-end pores (e.g., Skagius and Neretnieks 1986) but also open pores (e.g., Pérez-Rosales 1982).

The non-sorbing tracer diffuses in the fluid-saturated percolated pore driven by the local concentration gradient (Fick's first law). Assuming steady-state diffusion, the concentration obeys the 3D Laplace equation with a no-flux boundary condition on the solid/pore interface (e.g., Adler et al. 1992; Øren et al. 2007):

$$
\Delta C=0
$$


where $C$ is the tracer concentration in pore fluid.

The formation factor, $F$, refers to the electrical resistivity of porous rock/sediment saturated with a conductive fluid, normalized by that of the bulk fluid. It is well known that the formation factor obeys Archie's law (e.g., Schön 2004; Tiab and Donaldson 2004). Archie's law was originally developed for the electric current flowing in conductive pore fluid. The steady-state spatial distribution of the electric potential and tracer concentration in porous media obeys the same partial differential equation, i.e., the Laplace equation. Thus, it is possible to convert Archie's law for electrical resistivity into that for diffusivity as follows:

$$
F=\frac{D_{\text {bulk }}}{D_{\text {eff }}} \propto \phi^{-m}
$$

where $D_{\text {eff }}$ is the effective diffusivity, $D_{\text {bulk }}$ is the tracer diffusivity in bulk fluid, and $m$ is the exponent (e.g., Grathwohl 1998; Boving and Grathwohl 2001).

There is another important diffusivity, apparent diffusivity, $D_{\text {app }}$. The normalized apparent diffusivity is the reciprocal of the tortuosity of the pore structure of the porous media and obeys a power law similar to Archie's law:

$$
\text { normalized apparent diffusivity }=\frac{D_{\text {app }}}{D_{\text {bulk }}} \propto \phi^{n}
$$

where $n$ is the exponent (e.g., Nakashima 1995; Tiab and Donaldson 2004). The exponents, $m$ and $n$, were computed in the present study by the least squares method using the diffusion and porosity data. The exponents are sensitive to the degree of the rock deformation (e.g., Sen et al. 1981; Schön 2004).

The relationship between the apparent diffusivity and effective diffusivity is simple for non-sorbing tracer: $D_{\text {app }}=D_{\text {eff }} / \phi$ (e.g., Nakashima 1995). Substituting this expression into Eq. (A4) and comparing the result with Eq. (A3), we obtain the following:

$$
n=m-1
$$

\section{References}

Adler, P.M., Jacquin, C.G., Thovert, J.F.: The formation factor of reconstructed porous media. Water Resour. Res. 28, 1571-1576 (1992)

Andrade, J.S. Jr.., Almeida, M.P., Mendes Filho, J., Havlin, S., Suki, B., Stanley, H.E.: Fluid flow through porous media: the role of stagnant zones. Phys. Rev. Lett. 79, 3901-3904 (1997)

Boving, T.B., Grathwohl, P.: Tracer diffusion coefficients in sedimentary rocks: Correlation to porosity and hydraulic conductivity. J. Contam. Hydrol. 53, 85-100 (2001)

Cenedese, A., Viotti, P.: Lagrangian analysis of nonreactive pollutant dispersion in porous media by means of the particle image velocimetry technique. Water Resour. Res. 32, 2329-2343 (1996)

de Arcangelis, L., Redner, S., Coniglio, A.: Anomalous voltage distribution of random resistor networks and a new model for the backbone at the percolation threshold. Phys. Rev. B 31, 4725-4727 (1985)

Grathwohl, P.: Diffusion in Natural Porous Media: Contaminant Transport, Sorption/Desorption and Dissolution Kinetics. Kluwer, London (1998)

Han, M., Youssef, S., Rosenberg, E., Fleury, M., Levitz, P.: Deviation from Archie's law in partially saturated porous media: wetting film versus disconnectedness of the conducting phase. Phys. Rev. E 79, article number 031127 (2009)

Knackstedt, M.A., Arns, C.H., Sheppard, A.P., Senden, T.J., Sok, R.M., Cinar, Y., Olafuyi, A.O., Pinczewski, W.V., Padhy, G., Ioannidis, M.: Pore scale analysis of electrical resistivity in complex core material. In: International Symposium of the Society of Core Analysts, Calgary, SCA2007-33 (2007)

Kutsovsky, Y.E., Scriven, L.E., DavisH.T. Hammer, B.E.: NMR imaging of velocity profiles and velocity distributions in bead packs. Phys. Fluids 8, 863-871 (1996)

Lebon, L., Oger, L., Leblond, J., Hulin, J.P., Martys, N.S., Schwartz, L.M.: Pulsed gradient NMR measurements and numerical simulation of flow velocity distribution in sphere packings. Phys. Fluids 8, 293-301 (1996) 
Lever, D.A., Bradbury, M.H., Hemingway, S.J.: The effect of dead-end porosity on rock-matrix diffusion. J. Hydrol. 80, 45-76 (1985)

Li, X., Li, Z., Zhang, D.: Role of low flow and backward flow zones on colloid transport in pore structures derived from real porous media. Environ. Sci. Technol. 44, 4936-4942 (2010)

Lindquist, W.B., Venkatarangan, A., Dunsmuir, J., Wong, T.-F.: Pore and throat size distributions measured from synchrotron X-ray tomographic images of Fontainbleau sandstones. J. Geophys. Res $\mathbf{1 0 5}$, 21509-21527 (2000)

Maier, R.S., Kroll, D.M., Kutsovsky, Y.E., Davis, H.T., Bernard, R.S.: Simulation of flow through bead packs using the lattice Boltzmann method. Phys. Fluids 10, 60-74 (1998)

Nakano, T.: Numerical flow simulation through a two-dimensional network using X-ray CT imagery. Bull. Geol. Surv. Jpn. 46, 605-627 (1995) (In Japanese with English abstract)

Nakashima, S.: Diffusivity of ions in pore water as a quantitative basis for rock deformation rate estimates. Tectonophysics 245, 185-203 (1995)

Nakashima, Y., Kamiya, S.: Mathematica programs for the analysis of three-dimensional pore connectivity and anisotropic tortuosity of porous rocks using X-ray computed tomography image data. J. Nucl. Sci. Technol. 44, 1233-1247 (2007)

Nakashima, Y., Kamiya, S.: Anisotropic diffusion in fibrous porous media. J. Porous Media 13, 1-11 (2010)

Nakashima, Y., Nakano, T.: Accuracy of formation factors for three-dimensional pore-scale images of geomaterials estimated by renormalization technique. J. Appl. Geophys. 75, 31-41 (2011)

Nakashima, Y., Nakano, T., Nakamura, K., Uesugi, K., Tsuchiyama, A., Ikeda, S.: Three-dimensional diffusion of non-sorbing species in porous sandstone: computer simulation based on X-ray microtomography using synchrotron radiation. J. Contam. Hydrol. 74, 253-264 (2004)

Nakashima, Y., Kamiya, S., Nakano, T.: Diffusion ellipsoids of anisotropic porous rocks calculated by X-ray computed tomography-based random walk simulations. Water Resour. Res. 44, article number W12435 (2008)

Ogawa, K., Matsuka, T., Hirai, S., Okazaki, K.: Three-dimensional velocity measurement of complex interstitial flows through water-saturated porous media by the tagging method in the MRI technique. Meas. Sci. Technol. 12, 172-180 (2001)

Øren, P.-E., Bakke, S., Held, R.: Direct pore-scale computation of material and transport properties for North Sea reservoir rocks. Water Resour. Res. 43, article number W12S04 (2007)

Pérez-Rosales, C.: On the relationship between formation factor and porosity. Soc. Petrol. Eng. J. 22, 531-536 (1982)

Promentilla, M.A.B., Sugiyama, T., Hitomi, T., Takeda, N.: Quantification of tortuosity in hardened cement pastes using synchrotron-based X-ray computed microtomography. Cem. Concr. Res. 39, 548-557 (2009)

Roberts, J.N., Schwartz, L.M.: Grain consolidation and electrical conductivity in porous media. Phys. Rev. B 31, 5990-5997 (1985)

Schön, J.H.: Physical Properties of Rocks: Fundamentals and Principles of Petrophysics. Elsevier, Amsterdam (2004)

Sen, P.N., Scala, C., Cohen, M.H.: A self-similar model for sedimentary rocks with application to the dielectric constant of fused glass beads. Geophysics 46, 781-795 (1981)

Skagius, K., Neretnieks, I.: Porosities and diffusivities of some nonsorbing species in crystalline rocks. Water Resour. Res. 22, 389-398 (1986)

Sugiyama, T., Promentilla, M.A.B., Hitomi, T., Takeda, N.: Application of synchrotron microtomography for pore structure characterization of deteriorated cementitious materials due to leaching. Cem. Concr. Res. 40, 1265-1270 (2010)

Takeda, M., Hiratsuka, T., Ito, K., Finsterle, S.: An axisymmetric diffusion experiment for the determination of diffusion and sorption coefficients of rock samples. J. Contam. Hydrol. 123, 114-129 (2011)

Tiab, D., Donaldson, E.C.: Petrophysics: Theory and Practice of Measuring Reservoir Rock and Fluid Transport Properties, 2nd edn. Elsevier, Amsterdam (2004)

Watanabe, T., Katagishi, Y.: Deviation of linear relation between streaming potential and pore fluid pressure difference in granular material at relatively high Reynolds numbers. Earth Planets Space 58, 1045-1051 (2006)

Zhan, X., Schwartz, L.M., Toksöz, M.N., Smith, W.C., Morgan, F.D.: Pore-scale modeling of electrical and fluid transport in Berea sandstone. Geophysics 75, F135-F142 (2010)

Zhang, X., Knackstedt, M.A.: Direct simulation of electrical and hydraulic tortuosity in porous solids. Geophys. Res. Lett. 22, 2333-2336 (1995) 